\title{
Measurement of Diffractive Scattering of Photons with Large Momentum Transfer at HERA
}

\author{
Tomáš Hreus (On behalf of the H1 Collaboration) \\ Université Libre de Bruxelles - I.I.H.E. \\ Boulevard du Triomphe, CP230, 1050 Bruxelles, Belgium
}

\begin{abstract}
The first measurement of diffractive scattering of quasi-real photons with large momentum transfer $\gamma p \rightarrow \gamma Y$, where $Y$ is the proton dissociative system, is made using the H1 detector at HERA. Single differential cross sections are measured as a function of $W$, the incident photon-proton centre of mass energy, and $t$, the square of the fourmomentum transferred at the proton vertex. The $W$ dependence is well described by a perturbative QCD model using a leading logarithmic approximation of the BFKL evolution, whereas the measured $|t|$ dependence is harder than predicted.
\end{abstract}

\section{Introduction}

The study at the $e p$ collider HERA of exclusive diffractive processes in the presence of a hard scale provides insight into the parton dynamics of the diffractive exchange. The four-momentum squared transferred at the proton vertex, $t$, provides a relevant scale to investigate the application of perturbative Quantum Chromodynamics (pQCD) for $|t| \gg \Lambda_{\mathrm{QCD}}^{2}$ [2]. The diffractive photonproton scattering, $\gamma p \rightarrow \gamma Y$ at large $|t|$ gives an experimentally clean and almost fully perturbatively calculable process to study the parton dynamics. Its cross section measurement [3] is performed at HERA by studying the reaction $e^{+} p \rightarrow e^{+} \gamma Y$ in the

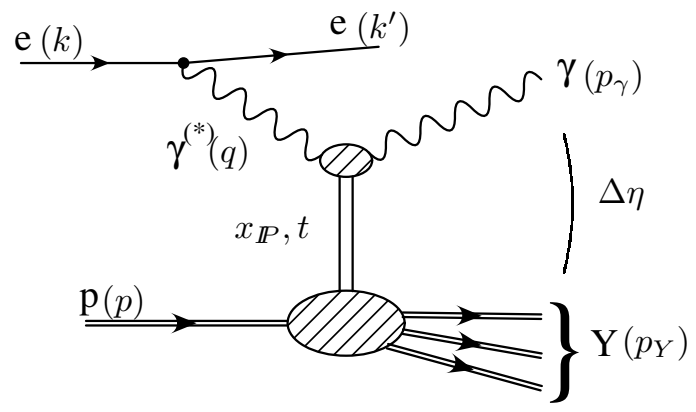

Figure 1: Schematic illustration of the $e p \rightarrow$ $e \gamma Y$ process.

photoproduction regime (initial photon virtualities $Q^{2}<0.01 \mathrm{GeV}^{2}$ ) and at $4<|t|<36 \mathrm{GeV}^{2}$, with a large rapidity gap between the final state photon and the proton dissociative system $Y$ (as illustrated in Fig. 1) and the $\gamma p$ centre of mass energy in the range $175<W<247 \mathrm{GeV}$.

Diffractive photon scattering can be modelled in the proton rest frame by the fluctuation of the incoming photon into a $q \bar{q}$ pair, which is then involved in a hard interaction with the proton via the exchange of a colour singlet state. In the leading logarithmic approximation (LLA), the colour singlet exchange is modelled by the effective exchange of a gluon ladder (Fig. 2), described at sufficiently low values of Bjorken $x$ by the BFKL [4] approach. In the LLA BFKL model the gluon ladder couples to a single parton within the proton. Due to the quasi-real nature of the incoming photon, the transverse momentum of the final state photon, $P_{T}^{\gamma}$, is entirely transferred by the gluon ladder to the parton in the proton. The separation in rapidity between the parton scattered by

DIS 2009 
the gluon ladder and the final state photon is given by $\Delta \eta \simeq \log \left(\hat{s} /\left(P_{T}^{\gamma}\right)^{2}\right)$, where $\hat{s}$ is the invariant mass of the system formed by the incoming photon and the struck parton.

In addition to the usual DIS kinematic variables, the longitudinal momentum fraction of the diffractive exchange with respect to the proton, $x_{\mathbb{P}}$ and the elasticity of the $\gamma p$ interaction, $y_{\mathbb{P}}$, are defined as (Following the notation in Fig. 1)

$$
x_{\mathbb{P}}=\frac{q \cdot\left(p-p_{Y}\right)}{q \cdot p}, \quad y_{\mathbb{P}}=\frac{p \cdot\left(q-p_{\gamma}\right)}{p \cdot q} .
$$

\section{Experimental procedure}

The data, corresponding to an integrated luminosity of $46.2 \mathrm{pb}^{-1}$, were collected with the H1 detector during the $1999-2000$ running period, when positrons of energy

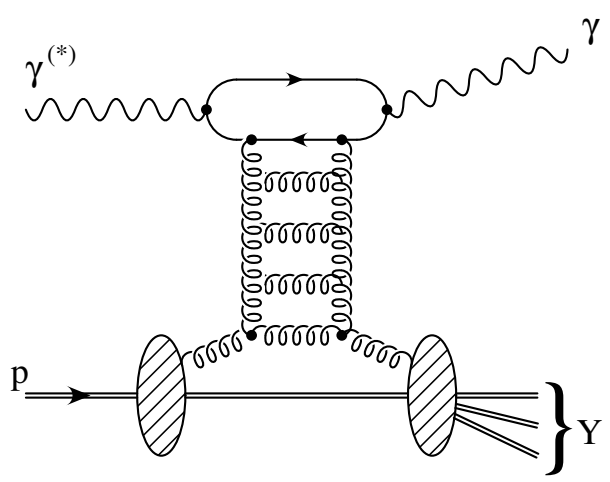
$E_{e}=27.6 \mathrm{GeV}$ collided with protons of Figure 2: Illustration of the $\gamma^{(*)} p \rightarrow \gamma Y$ process in a LLA BFKL approach. energy $E_{p}=920 \mathrm{GeV}$. The detailed description of the $\mathrm{H} 1$ detector can be found in [5]. The quantity $W$ is calculated from the measured energy of the scattered positron, $E_{e^{\prime}}$, using the relation $W^{2} \simeq y s \simeq s\left(1-E_{e^{\prime}} / E_{e}\right)$ with the relative resolution $4 \%$, where $s=(k+p)^{2}$ is the $e p$ centre of mass energy squared. The scattered positron is detected in a small calorimeter $33 \mathrm{~m}$ downstream the main detector. The photon candidate is detected as a cluster in the electromagnetic section of the calorimeter, with no associated track and is furthermore required to have an energy $E_{\gamma}>8 \mathrm{GeV}$ and a transverse momentum $P_{T}^{\gamma}>2 \mathrm{GeV}$. The kinematic variable $|t|$ is reconstructed as $|t| \simeq\left(P_{T}^{\gamma}\right)^{2}$, with the resolution of $11 \%$. The hadronic final state $Y$ is reconstructed using a combination of tracking and calorimetric information. The event inelasticity of the $\gamma p$ interaction is reconstructed as $y_{\mathbb{P}} \simeq \sum_{Y}\left(E-P_{z}\right) /\left[2\left(E_{e}-E_{e^{\prime}}\right)\right]$, where the summation is performed on all detected hadronic final state particles in the event. Diffractive events are selected by requiring $y_{\mathbb{P}}<0.05$, ensuring a large pseudorapidity gap, $\Delta \eta$, between the photon and the proton dissociative system $Y$, since $y_{\mathbb{P}} \simeq e^{-\Delta \eta}$.

After applying all selection criteria, described in a more detail in $[3,6], 240$ events remain in the data sample.

\section{Models}

The HERWIG 6.4 MC event generator is used to simulate the diffractive high $|t|$ photon scattering using the LLA BFKL model $[7,8,9]$. At leading logarithmic accuracy there are two independent free parameters in the BFKL calculation: the value of the strong coupling $\alpha_{s}$ and the scale, $c$, which defines the leading logarithms in the expansion of the BFKL amplitude, $\ln \left(W^{2} /(c|t|)\right)$. In exclusive production of vector mesons, the scale parameter $c$ is related to the vector meson mass. In the case of diffractive photon scattering, the unknown scale results in the absence of a normalisation prediction of the cross section. In 
the calculations considered here, the running of $\alpha_{s}$ as a function of the scale is ignored and will henceforth be referred to as $\alpha_{s}^{B F K L}$. The choice of $\alpha_{s}^{B F K L}=0.17$ is used for the simulation for this measurement.

In the asymptotic approximation of the calculations [9], the $\mathrm{W}$ dependence of the cross section follows a power-law, $\sigma(W) \sim W^{4 \omega_{0}}$, where the exponent is given by the choice of $\alpha_{s}^{B F K L}$ by $\omega_{0}=\left(3 \alpha_{s}^{B F K L} / \pi\right) 4 \ln 2$. For the $t$ dependence of the cross section, an approximate power-law behaviour is predicted of the form $\mathrm{d} \sigma / \mathrm{d}|t| \sim|t|^{-n}$, where $n$ depends on the parton density functions of the proton and on the value of $\alpha_{s}^{B F K L}$. In order to describe the data, the $t$ dependence of the diffractive photon scattering simulation was weighted by a factor $|t|^{0.73}$. This weighted HERWIG prediction is used only to correct the data for resolution and acceptance effects.

Possible sources of background were estimated using MC simulations and two of these sources were identified as non-negligible. The background from inclusive diffractive photoproduction events $(e p \rightarrow e X Y$, where the two hadronic final states are separated by a rapidity gap) is simulated using the PHOJET MC event generator. This background contributes when a single electromagnetic particle fakes the photon candidate and the process is estimated to amount to $3 \%$ of the measured cross section. The background from electron pair production $\left(e p \rightarrow e e^{+} e^{-} X\right)$ is modelled using the GRAPE event generator. This process contributes to the selection if two of the leptons fake signals from the photon candidate and the scattered electron, whereas the remaining lepton escapes detection. This background contributes $4 \%$ of the measured cross section.

\section{Cross section measurement}

The $e p \rightarrow e \gamma Y$ differential cross sections are defined using the formula:

$$
\frac{\mathrm{d}^{2} \sigma_{e p \rightarrow e \gamma Y}}{\mathrm{~d} W \mathrm{~d} t}=\frac{N_{d a t a}-N_{b g r}}{\mathcal{L} A \Delta W \Delta t}
$$

where $N_{d a t a}$ is the number of observed events corrected for trigger efficiency, $N_{b g r}$ the expected contribution from background events, $\mathcal{L}$ the integrated luminosity, $A$ the signal acceptance and $\Delta W$ and $\Delta t$ the bin widths in $W$ and $t$, respectively. The $\gamma p \rightarrow \gamma Y$ differential cross section is then extracted from the $e p$ cross section using:

$$
\frac{\mathrm{d}^{2} \sigma_{e p \rightarrow e \gamma Y}}{\mathrm{~d} W \mathrm{~d} t}=\Gamma(W) \frac{\mathrm{d} \sigma_{\gamma p \rightarrow \gamma Y}}{\mathrm{~d} t}(W),
$$

where the photon flux, $\Gamma(W)$, is integrated over the range $Q^{2}<0.01 \mathrm{GeV}^{2}$ according to the modified Weizsäcker-Williams approximation [10]. The $\gamma p$ cross section is obtained by modelling $\sigma_{\gamma p \rightarrow \gamma Y}$ as a power-law in $W$, whose parameters are iteratively adjusted to reproduce the measured $W$ dependence of the $e p$ cross section. The differential $\gamma p$ cross section in $|t|$ is then extracted from the ep cross section by correcting for the effect of the photon flux over the visible $W$ range. The $\gamma p$ cross section as a function of $W$ is obtained by first integrating the above equation over the $|t|$ range, and then correcting for the effect of the photon flux in each bin in $W$.

The systematic error on the measurement stems from experimental uncertainties and from model dependences. They are calculated using the weighted HERWIG simulation of the signal process. The uncertainty on the PHOJET MC normalisation and the size of the

DIS 2009 

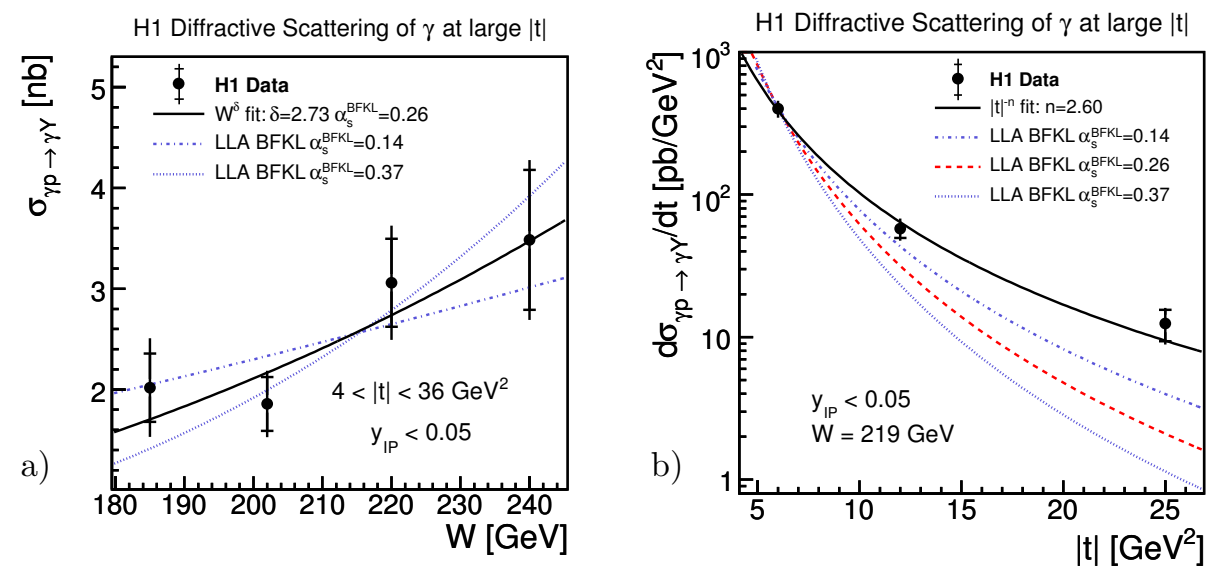

Figure 3: The $\gamma p$ cross section of diffractive scattering of photons as a function of a) $W$, b) $|t|$. The solid line represents a fit to the cross section. Additional curves show the LLA BFKL predictions corresponding to different $\alpha_{s}^{B F K L}$.

variation of the model dependence on $x_{\mathbb{P}},|t|$ and $M_{Y}$, are estimated from the measured distributions in the data: the variation corresponds to the a range where the model still describes the data. Each source of systematic error is varied in the weighted HERWIG Monte Carlo within its uncertainty. The largest systematic error on the cross sections comes from the uncertainty on the $x_{\mathbb{P}}$ and $M_{Y}$ dependences. The total systematic error on the $W$ and $|t|$ dependence of the cross section varies from $10 \%$ to $17 \%$ and from $8 \%$ to $14 \%$, respectively. An additional global uncertainty of $4 \%$ arises from the $\gamma p$ cross section extraction procedure. The total systematic errors are comparable to or smaller than the statistical errors.

\section{$5 \quad$ Results and conclusions}

The cross sections are measured in the domain $175<W<247 \mathrm{GeV}, 4<|t|<36 \mathrm{GeV}^{2}$, $y_{\mathbb{P}}<0.05$ and $Q^{2}<0.01 \mathrm{GeV}^{2}$. The $\gamma p \rightarrow \gamma Y$ cross section as a function of $W$ is shown in Fig. 3a. A power-law dependence of the form $\sigma \sim W^{\delta}$ is fitted to the measured cross section, the fit yields $\delta=2.73 \pm 1.02$ (stat.) ${ }_{-0.78}^{+0.56}$ (syst.) with $\chi^{2} /$ n.d.f. $=2.7 / 2$. The steep rise of the cross section with $W$ is usually interpreted as an indication of the presence of a hard sub-process in the diffractive interaction and of the applicability of perturbative QCD. The present $\delta$ value, measured at an average $|t|$ value of $6.1 \mathrm{GeV}^{2}$, is compatible with that measured by $\mathrm{H} 1$ in diffractive $J / \psi$ photoproduction of $\delta=1.29 \pm 0.23$ (stat.) \pm 0.16 (syst.) [11] at an average $|t|$ of $6.9 \mathrm{GeV}^{2}$. The Pomeron intercepts associated with these $\delta$ values correspond to the strongest energy dependences measured in diffractive processes.

The $\gamma p$ cross section differential in $|t|$, at $W=219 \mathrm{GeV}$, is shown in Fig. 3b, together with a fit of the form $\mathrm{d} \sigma / \mathrm{d} t \sim|t|^{-n}$, where the fitted $n=2.60 \pm 0.19$ (stat.) ${ }_{-0.08}^{+0.03}$ (syst.) with $\chi^{2} /$ n.d.f. $=1.6 / 1$. The $|t|$ dependence is harder than that measured by H1 in the diffractive photoproduction of $J / \psi$ mesons at large $|t|$ [11]. The measured cross sections are

DIS 2009 
compared to predictions of the LLA BFKL model, using the HERWIG Monte Carlo with no $|t|$ weighting. The predictions are normalised to the integrated measured cross section, as the normalisation is not predicted by the LLA BFKL calculation. The $W$ dependence of the cross section is well described by the LLA BFKL prediction. Using $\delta=4 \omega_{0}=4\left(3 \alpha_{S}^{F i t} / \pi\right) 4 \ln 2$, the measured $W$ dependence leads to $\alpha_{S}^{F i t}=0.26 \pm 0.10$ (stat. $)_{-0.07}^{+0.05}$ (syst.). Predictions are shown in Figure 3 also for the values $\alpha_{s}^{B F K L}=0.14$ and 0.37, corresponding to one standard deviation of $\alpha_{S}^{F i t}$.

As shown in Figure 3b, the LLA BFKL calculation for $\alpha_{s}^{B F K L}=0.14,0.26$ and 0.37 , all of which give a reasonable description of the $W$ dependence, predict steeper $|t|$ distributions than is measured in the data. The same effect cannot be established for the exclusive $\rho$ measurement [12], where the measured $t$ range is limited to $|t|<8 \mathrm{GeV}^{2}$, although an underestimate of the cross section was observed at the largest values of $|t|$. The present situation is in contrast with the analysis of $J / \psi$ production $[11,13]$, where the $|t|$ dependence was found to be well described by the LLA BFKL prediction over a similar range in $t$.

\section{References}

[1] Slides: http://indico.cern. ch/contributionDisplay $\cdot$ py? contribId=172\&sessionId=18\&conf $I d=53294$

[2] J. R. Forshaw and P. J. Sutton, Eur. Phys. J. C 1 (1998) 285 [arXiv:hep-ph/9703225].

[3] F. D. Aaron et al. [H1 Collaboration], Phys. Lett. B 672 (2009) 219 [arXiv:0810.3096 [hep-ex]].

[4] E. A. Kuraev, L. N. Lipatov and V. S. Fadin, Sov. Phys. JETP 45 (1977) 199 [Zh. Eksp. Teor. Fiz. 72 (1977) 377].

[5] I. Abt et al. [H1 Collaboration], Nucl. Instrum. Meth. A 386 (1997) 310.

[6] T. Hreus, PhD Thesis, Université Libre de Bruxelles and Pavol Jozef Šafárik University (2008), available at http://www-h1.desy.de/psfiles/theses/h1th-495.pdf

[7] D. Y. Ivanov and M. Wusthoff, Eur. Phys. J. C 8 (1999) 107 [arXiv:hep-ph/9808455].

[8] N. G. Evanson and J. R. Forshaw, Phys. Rev. D 60 (1999) 034016 [arXiv:hep-ph/9902481].

[9] B. E. Cox and J. R. Forshaw, J. Phys. G 26 (2000) 702 [arXiv:hep-ph/9912486].

[10] S. Frixione, M. L. Mangano, P. Nason and G. Ridolfi, Phys. Lett. B 319 (1993) 339 [arXiv:hep$\mathrm{ph} / 9310350]$.

[11] A. Aktas et al. [H1 Collaboration], Phys. Lett. B 568 (2003) 205 [arXiv:hep-ex/0306013].

[12] A. Aktas et al. [H1 Collaboration], Phys. Lett. B 638 (2006) 422 [arXiv:hep-ex/0603038].

[13] S. Chekanov et al. [ZEUS Collaboration], Eur. Phys. J. C 26 (2003) 389 [arXiv:hep-ex/0205081]. 